\title{
Effect of leptin on spatial learning, memory and blood glucose level in streptozotocin induced diabetes mellitus in male wistar albino rats
}

\author{
Archita C. Joshi, Chetna R. Patel*, Naresh D. Kantharia
}

Department of Pharmacology, Government Medical College, Surat, Gujarat, India

\author{
Received: 18 November 2019 \\ Revised: 03 December 2019 \\ Accepted: 04 December 2019 \\ *Correspondence: \\ Dr. Chetna R. Patel, \\ Email: drchetnapate12012@yahoo.com
}

Copyright: (c) the author(s), publisher and licensee Medip Academy. This is an open-access article distributed under the terms of the Creative Commons Attribution Non-Commercial License, which permits unrestricted non-commercial use, distribution, and reproduction in any medium, provided the original work is properly cited.

\begin{abstract}
Background: Diabetes mellitus is known to cause cognitive impairment that can be possibly attributed to deficient levels of leptin in diabetic animals. This study was undertaken to study the effect of administration of leptin on spatial learning, memory and blood glucose levels in diabetic rats.

Methods: Rats were divided into three groups. The first group was the control group. Diabetes was induced in groups 2 and 3 by streptozotocin (STZ) injection $(60 \mathrm{mg} / \mathrm{kg}$ ) intraperitoneally. Group 2 received saline while group 3 received leptin $(0.1 \mathrm{mg} / \mathrm{kg})$ subcutaneously for 10 days from $4^{\text {th }}$ day of STZ administration. Behavioural assessment was done in $\mathrm{T}$ maze after 21 days of the last injection of leptin. Blood glucose levels were also analysed.

Results: The number of correct arm entries decreased while time spent being immobile and time spent to reach the correct arm increased in the diabetic group when compared to the control group and correct arm entries increased while time spent immobile and time spent to reach the correct arm decreased with leptin treatment when compared to the diabetic control rats. Blood glucose levels increased in the diabetic rats while leptin administration reduced blood glucose levels in the group 3.

Conclusions: Our study suggests that leptin can improve learning and memory while also producing a slight reduction in the blood glucose levels in diabetic rats.
\end{abstract}

Keywords: Leptin, Streptozotocin, Cognition, T maze, Diabetes mellitus

\section{INTRODUCTION}

Diabetes mellitus (DM) is fast gaining the status of potential epidemic in India with more than 62 million diabetic individuals currently diagnosed with the disease. ${ }^{1}$ About 422 million people are diabetics worldwide. In 2012 , diabetes was the direct cause of 1.5 million deaths. ${ }^{2}$ It is associated with reduced life expectancy, significant morbidity due to microvascular complications, macrovascular complications and diminished quality of life. ${ }^{3}$

DM is associated with cognitive deficits and an increased risk of dementia, particularly in the elderly. These deficits are paralleled by neurophysiological and structural changes in the brain. In animal models of diabetes, impairments of spatial learning occur in association with distinct changes in hippocampal synaptic plasticity. At the molecular level these impairments might involve changes in glutamate-receptor subtypes, in secondmessenger systems and in protein kinases. The multifactorial pathogenesis of diabetic encephalopathy is not yet completely understood, but clearly shares features with brain ageing and the pathogenesis of diabetic neuropathy. It involves both metabolic and vascular changes, related to chronic hyperglycaemia but probably also defects in insulin action in the brain. ${ }^{4}$ 
Recently, the association of diabetes with dementia and cognitive dysfunction has gained attention. ${ }^{5}$ Patients with DM are 1.5 times more likely to develop Alzheimer's disease and 2.5 times more likely to develop vascular dementia than those without diabetes. ${ }^{6}$ In patients with type 1 diabetes, changes are seen in the first five to seven years of life when the brain is developing. Studies in adults showed that a specific set of cognitive functions is affected by type 1 DM, including mental processing speed and flexibility and attention. Other important functions, such as memory and learning, planning and inhibition seem to be unaffected in these patients. ${ }^{7}$ The profile of cognitive decrements in type $2 \mathrm{DM}$ is generally similar to that found in type $1 \mathrm{DM}^{8}$ The most striking difference is that type $2 \mathrm{DM}$ is associated with decreased memory and learning performance and vascular brain lesions are more prominent. ${ }^{9}$

It is well documented that leptin plays a pivotal role in the regulation of food intake and body weight via signalling information about the status of fat stores to leptin receptors expressed on specific hypothalamic nuclei. Studies have shown that leptin plays an important role in modulating higher cognitive functions such as learning and memory and that leptin is a potential cognitive enhancer as it rapidly alters glutamate receptor trafficking processes and in turn the efficacy of hippocampal excitatory synaptic transmission.

It is well documented that cognitive deficits are prevalent in diabetics. As obesity and obesity-linked disorders such as type II diabetes are associated with resistance to leptin, it is feasible that leptin dysfunction plays a role in cognitive impairments in these individuals. ${ }^{10}$ However it was found that when type 1 diabetic children are insulin deficient, which is classically the case at initial presentation, their leptin levels are subnormal to a control population. Furthermore, their leptin levels do not correlate with BMI, indicating that factors besides adiposity are operational. Soon after the institution of insulin therapy (within $24 \mathrm{hr}$ ), leptin levels increase significantly before any appreciable weight gain. These levels become comparable to non-diabetic levels after 3-5 days of insulinization. At that point, leptin levels correlate with insulin dose. These results suggest a stimulatory role of insulin on leptin production. ${ }^{11}$ Due to the lack of adequate evidence on the relationship between leptin and its beneficial effects (if any) on impaired learning and memory in type 1 diabetes, we decided to conduct this experiment to study the effects of leptin on spatial learning, memory and blood glucose levels in streptozotocin induced diabetes mellitus in male albino wistar rats.

\section{METHODS}

\section{Experimental animals}

All experiments were performed after prior permission from the Institutional Animal Ethics Committee (IAEC),
Government Medical College, Surat, Gujarat, India. Committee is constituted in accordance with Committee for the Purpose of Control and Supervision on Experiments on Animals (CPCSEA) guidelines, Ministry of Environment and Forests (Animal Welfare Division), Government of India, New Delhi, India during 1 year period (November 2015 to October 2016). Male wistar albino rats (150-250 gms) were procured from the Central Animal House of Government Medical College, Surat.

Rats were housed in standard transparent polypropylene cages and kept under controlled room temperature and humidity $\left(26 \pm 30{ }^{\circ} \mathrm{C} ; 40 \pm 5 \%\right)$ in a $12 \mathrm{hr}$ light-dark cycle. Animals were acclimatized for one week to laboratory conditions before starting the experiment. The rats were given standard laboratory diet and water ad libitum.

Prior to the day of initiation of the experiment, the rats were fasted for 12 hours with free access to water. Day 1 is taken as the day of beginning of the study.

\section{Drugs}

Streptozotocin: Streptozotocin was purchased in powder form from Venus Chemicals Centre, Vapi. It was dissolved in normal saline $(100 \mathrm{mg} / 5 \mathrm{ml})$ and administered in a dose of $60 \mathrm{mg} / \mathrm{kg}$ of body weight intraperitoneally (I.P.).

Leptin: Leptin (recombinant rat leptin) was purchased in powder form from Labdhee Laboratories, Ahmedabad. It was dissolved in distilled water to get the concentration of $1 \mathrm{mg} / \mathrm{ml}$ and was given to the rats in a dose of 0.1 $\mathrm{mg} / \mathrm{kg}$ of body weight subcutaneously (S.C.). ${ }^{12}$

\section{Induction of diabetes}

Diabetes was induced in the rats by injecting streptozotocin in the dose of $60 \mathrm{mg} / \mathrm{kg}$ body weight, I.P., in all the 3 groups. It was injected once, on day 1.

To assess if diabetes had been successfully induced, blood glucose analysis was done by a glucometer, 72 hours after the injection.

\section{Experimental design}

There were a total of 3 groups in the study with each group containing 5 animals. 15 rats were allocated randomly into each group.

Group 1 (control group): This group consisted of healthy rats which received no treatment.

Group 2 (diabetic rats receiving normal saline): The rats in this group were given streptozotocin $60 \mathrm{mg} / \mathrm{kg}$ I.P. on the first day of conduction of the experiment. After checking for successful induction of diabetes, normal saline in the dose of $0.1 \mathrm{mg} / \mathrm{kg}$ was administered S.C. 
from the $4^{\text {th }}$ day (of streptozotocin injection) onwards, for 10 consecutive days. The injection sites were alternatively changed (interchanging between the right and left hip).

Table 1: Experimental design.

\begin{tabular}{|c|c|c|c|}
\hline & Treatment received (day 1$)$ & Treatment received ( day 4) & Behavioral assessment (day 14) \\
\hline Group 1 & No treatment & No treatment & \multirow{3}{*}{$\begin{array}{l}\text { Behavioural assessment was done on the } \mathrm{T} \\
\text { maze after } 4 \text { weeks of streptozotocin } \\
\text { injection. }\end{array}$} \\
\hline Group 2 & $\begin{array}{l}\text { Streptozotocin } 60 \mathrm{mg} / \mathrm{kg} \text { I.P. } \\
\text { once. }\end{array}$ & Normal saline $0.1 \mathrm{mg} / \mathrm{kg} \mathrm{S.C.}$ & \\
\hline Group 3 & $\begin{array}{l}\text { Streptozotocin } 60 \mathrm{mg} / \mathrm{kg} \text { I.P. } \\
\text { once. }\end{array}$ & Leptin $0.1 \mathrm{mg} / \mathrm{kg} \mathrm{S.C.}$ & \\
\hline
\end{tabular}

Group 3 (diabetic rats receiving leptin): The rats in this group were given streptozotocin $60 \mathrm{mg} / \mathrm{kg}$ I.P. on the first day of conduction of the experiment. After checking for successful induction of diabetes, leptin in the dose of 0.1 $\mathrm{mg} / \mathrm{kg}$ was administered S.C. from the $4^{\text {th }}$ day (of streptozotocin injection) onwards, for 10 consecutive days. The injection sites were alternatively changed (interchanging between the right and left hip) (Table 1).

\section{Behavioural assessment}

Four weeks after the streptozotocin injection, behavioural assessment in terms of spatial learning and memory was done by using the $\mathrm{T}$ maze.

\section{Procedure}

Rats after the successful induction of diabetes were subjected to behavioural assessment in the $\mathrm{T}$ maze test after completing the required treatment lengths. ${ }^{12}$

\section{Habituation}

Initially the rats were made to run in the $\mathrm{T}$ maze for a period of 4 days so that they adapt to the $\mathrm{T}$ maze and can differentiate between the right and left sides. This habituation made them well versed with the maze and provided them with the orientation required for the future trials. Visual cues in the form of different shapes of a triangle and a cylinder were placed alongside both the arms to allow the rats to distinguish between the left and the right sides. White paper was placed in the floor of the maze which was removed after every trial so that the fecal matter and urine left behind by the rat during the trial doesn't guide their choice in the next trial.

\section{Main task}

After the habituation was complete, the main test procedure was carried out. Each rat had to undergo 20 trials spaced out on a total of 5 days. Every day, 2 sessions were conducted divided to take place in the morning and afternoon with each session comprising of 2 trials. Hence each day, a total of 4 trials occurred. Each trial comprised of 2 phases, phase I and phase II. Phase I was the phase assigned to learning. The rat was placed at the base of the $\mathrm{T}$. It was allowed to make a choice by selecting either the left or the right arm of the $\mathrm{T}$ maze. Whichever arm the rat chose, it was confined in it by placing the block at the end of the arm for a period of 30 seconds to help retain the information regarding the choice of side. After 30 seconds, the rats were removed from the blocked arms and placed in a cage outside the maze for 1 minute. The block was removed and the rat was again placed in the base of the $\mathrm{T}$ and allowed to run and make a choice.

A correct alternation was said to have been made when the rat chose the arm which was not previously chosen. The distance was measured from the base of the $\mathrm{T}$ to the entry point of the arm chosen and the task was said to be complete when the whole body along with the tail was inside of the arm chosen. The time taken to reach the arm, the time spent immobile and the speed of the rats were noted for each run.

Results were analysed by one way ANOVA followed by Tukey's post hoc test.

\section{Ethical approval}

All experiments were performed after prior permission from the Institutional Animal Ethics Committee (IAEC), Government Medical College, Surat, Gujarat, India. Committee is constituted in accordance with CPCSEA guidelines, Ministry of Environment and Forests (Animal Welfare Division), Government of India, New Delhi, India.

\section{RESULTS}

The purpose of this study was to determine if the administration of leptin to the group 3 rats caused a reduction in the blood glucose levels and an improvement in the cognitive functions when compared to the group 1 and group 2 rats. The behavioural assessment was done using the $\mathrm{T}$ maze and the rats were tested on the following parameters:

- Time taken to reach arm in both phases, phase I and phase II.

- Time spent immobile in both phases, phase I and phase II.

- $\quad$ Speed of the rat in both phases, phase I and phase II.

- Whether the rat alternated correctly or not. 
Apart from the parameters tested on the $\mathrm{T}$ maze, blood glucose analysis was also done to determine the effect of leptin on blood glucose. The results obtained are described below.

\section{Result of blood glucose analysis}

Streptozotocin administration to the group 2 and group 3 rats led to an increase in the blood glucose levels when compared to the group 1 rats. Leptin administration to the group 3 rats however brought about a decrease in the blood glucose levels when compared to the group 2 rats which received normal saline.

Leptin administration did not bring down the blood glucose levels to the levels of the control rats (group-1) however they were significantly lowered when compared to the levels of group 2 rats (Table 2).

Table 2: Blood glucose levels in $\mathrm{mg} / \mathrm{dl}$.

\begin{tabular}{|llll|}
\hline Group & Day 1 & Day 4 & Day 14 \\
\hline I (control) & $74 \pm 2.77$ & $72.6 \pm 2.87$ & $74.2 \pm 1.68$ \\
\hline $\begin{array}{l}\text { II (diabetic- } \\
\text { normal saline) }\end{array}$ & $73.6 \pm 3.64$ & $276.8 \pm 4.42$ & $284.8 \pm 6.61$ \\
\hline $\begin{array}{l}\text { III (diabetic- } \\
\text { leptin) }\end{array}$ & $72.2 \pm 3.98$ & $270.8 \pm 5.18$ & $236 \pm 9.06$ \\
\hline
\end{tabular}

Results are expressed as Mean \pm SEM ( $\mathrm{N}=5$ for each group). The readings are then analysed to assess a difference between the three groups. ANOVA followed by Tukey's post hoc test revealed a significant difference between the three groups $(\mathrm{p}<0.01)$.

\section{Results of the behavioural assessment}

The analysis done on the $\mathrm{T}$ maze was done to assess the cognition of the diabetic rats and compare it with the controls as well as with those rats that had received leptin. The assessment was done on the basis of the following parameters:

Results were analysed by one way ANOVA followed by Tukey's post hoc test. There was a statistically significant difference observed between the group 1 and group 2, as well as group 1 and group $3(\mathrm{p}<0.01)$. Statistically significant difference was also observed between the group 2 and group $3(\mathrm{p}<0.01)$ (Table 3$)$.

Table 3: Forced alternation rate.

\begin{tabular}{|ll|}
\hline Group & Mean \pm sem \\
\hline Group 1 & $84 \pm 2.915$ \\
\hline Group 2 & $57 \pm 4.062 *$ \\
\hline Group 3 & $65 \pm 1.581 * \#$ \\
\hline
\end{tabular}

$* \mathrm{P}<0.01$ as compared to control; $\# \mathrm{p}<0.01$ as compared to group 2 .

There is a statistically significant difference between the three groups with reference to the P1 using one way
ANOVA followed by Tukey's post hoc test. While the difference was statistically significant between group 1 versus group 2 and group 2 versus group $3(\mathrm{p}<0.01)$, difference was also observed between group 2 and group $3(\mathrm{p}<0.05)$ (Figure 1).

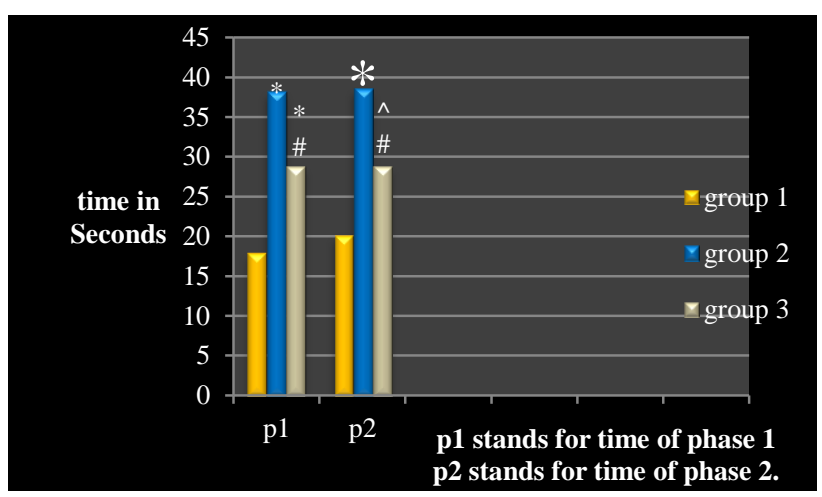

Figure 1: Time taken to reach the target arm. $* \mathrm{P}<0.01$ as compared to group $1 ; \# \mathrm{p}<0.05$ as compared to group $2 ;{ }^{\wedge} \mathrm{p}<0.05$ compared to group 1 .

In $\mathrm{P} 2$, statistically significant difference has been observed between the 3 groups using one way ANOVA followed by Tukey's post hoc test. Group 2 and group 3 were significantly different from the control rats at the $\mathrm{p}<0.01$ and 0.05 respectively. Group 2 differed from group 3 and the difference was significant $(\mathrm{p}<0.05)$.

There is a statistically significant difference between the three groups with reference to the i1 using one way ANOVA followed by Tukey's post hoc test. While the difference was statistically significant between group 1 versus group 2 and group 2 versus group $3(\mathrm{p}<0.01)$, difference was also observed between group 2 and group 3 at $(\mathrm{p}<0.01)$ (Figure 2).

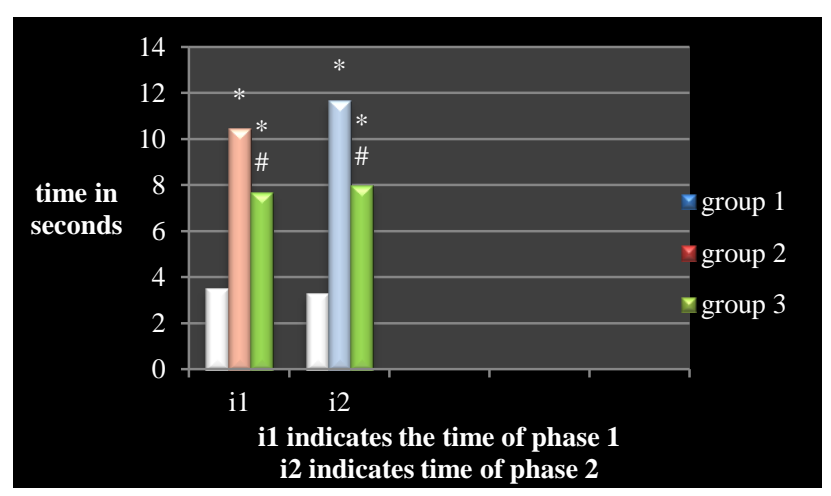

Figure 2: Time spent immobile while performing the task.

$* \mathrm{P}<0.01$ as compared to group $1 ; \# \mathrm{p}<0.01$ as compared to group 2.

In i2, statistically significant difference has been observed between the 3 groups using one way ANOVA followed by Tukey's post hoc test. Group 2 and group 3 were significantly different from the control rats $(\mathrm{p}<0.01)$. 
Group 2 differed from group 3 and the difference was significant $(\mathrm{p}<0.01)$.

There is a statistically significant difference between the three groups with reference to the s1 using one way ANOVA followed by Tukey's post hoc test. While the difference was statistically significant between group 1 versus group 2 and group 1 versus group $3(\mathrm{p}<0.05)$, no statistically significant difference was also observed between group 2 and group 3 (Figure 3).

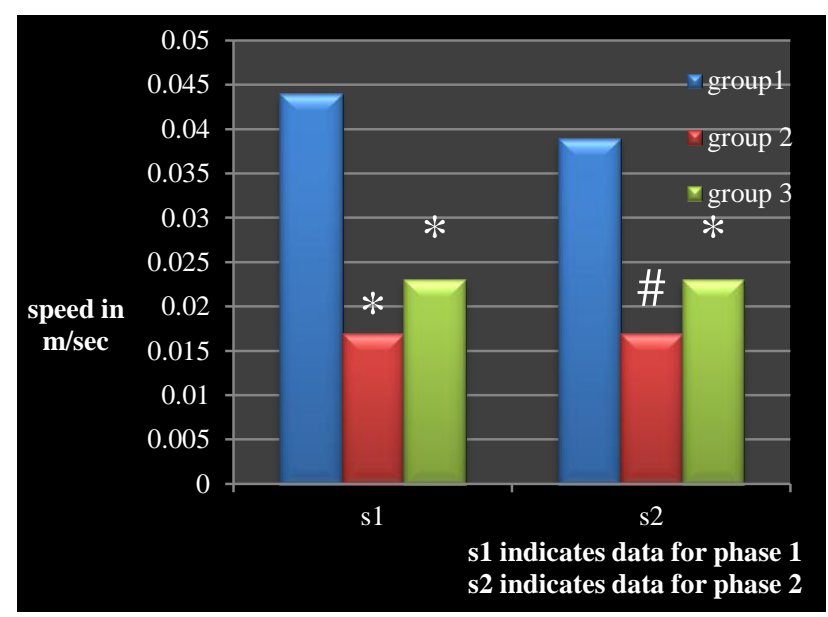

Figure 3: Speed of the rats while performing the task. $* \mathrm{P}<0.05$ with respect to the group $1 ; \# \mathrm{p}<0.01$ with respect to group 1 .

In s2, statistically significant difference has been observed between the 3 groups using one way ANOVA followed by Tukey's post hoc test. Group 1 and group 3 were significantly different from the control rats $(\mathrm{p}<0.05)$. Group 1 differed from group $2 \quad(\mathrm{p}<0.01)$ and no statistically significant difference was observed between the group 2 and group 3 in s 2 as well.

\section{DISCUSSION}

Cognitive dysfunction with its wide range, from mild cognitive impairment through dementia, is one of the chronic complications of diabetes mellitus. ${ }^{13}$ About $80 \%$ of people with Alzheimer's disease may have diabetes or impaired fasting glucose. ${ }^{14}$ There is a faster deterioration of cognition in diabetic patients rather than non-diabetic elderly ones. $^{15}$

Studies have shown that in patients with type 1 diabetes, cognitive dysfunction is characterized by a slowing of mental speed and a diminished mental flexibility. In some studies, sparing of learning and memory functions was reported, while some studies showed deficits in psychomotor efficiency and learning and memory. ${ }^{16,17}$ This heterogeneity is probably caused by differences in patient characteristics and the psychometric paradigms used. Consequently, the exact pattern and magnitude of cognitive dysfunction is still unclear. ${ }^{16}$
Leptin has multiple, complex actions on insulin-sensitive tissues and the hormones of the endocrine pancreas, all of which likely contribute to glucose homeostasis. Both leptin deficiency and leptin resistance have a profound impact on metabolism in rodents and humans. Leptin replacement can dramatically improve metabolism in cases of leptin deficiency, and now even shows promise as a therapy for insulin deficient type 1 diabetes. ${ }^{18}$

As a result of the profound ability of leptin to modulate glucose homeostasis, leptin holds therapeutic potential for the treatment of metabolic disorders. ${ }^{18}$ Clinical trials showed that leptin had modest weight-reducing effects in some obese individuals. ${ }^{19}$ One such metabolic disorder that is accompanied by inappropriately low leptin levels is type 1 diabetes.

Although insulin therapy restores circulating leptin levels in type 1 diabetes, studies in rodents show that higher doses of leptin might have additional therapeutic effects on glycemia. Leptin is now being tested clinically as an adjunct to insulin therapy for type 1 diabetic patients. ${ }^{20}$

In our study, the main focus was on the fasting blood sugar and the behavioural assessment done on the T maze which tested the animals on the time taken to reach the arms, the time spent immobile and the speed with which they reached their targets.

The assessment of blood glucose showed that there was a statistically significant increase in the blood glucose levels in the group 2 and group 3 after the administration of streptozotocin as checked on the $4^{\text {th }}$ day since the beginning of the experiment. The administration of leptin to the group 3 rats caused a decrease in the levels of glucose (levels measured on day 14) when compared to glucose levels of the same rats as seen on day 4 (post diabetes induction) as well as when compared to the glucose levels of the group 2 rats as seen on day 14 .

These results are in line with the findings of the study conducted by Ghasemi et al. ${ }^{12}$ Ghasemi and his colleagues in their study showed that leptin administration in the same dose as the one used in our study brought about a decrease in the levels of blood glucose in the rats. This decline in the blood glucose paralleled the improvement in cognition seen in their study.

This decrease in blood glucose levels by leptin may be explained by the fact there is a hypothesised crosstalk between the leptin and the insulin intracellular signalling pathways at the level of phosphatidylinositol-3 kinase (PI3-kinase). ${ }^{21}$ In skeletal muscle, the insulin effect on glucose uptake is mainly mediated by GLUT4 translocation. A central role of PI3-kinase, both in the signalling to GLUT4 translocation and glycogen synthesis, has been demonstrated. ${ }^{22,23}$ It is also in accordance with preliminary studies of skeletal muscle that demonstrated increased (up to 2-fold) GLUT4 expression in leptin-treated mice, which suggests that 
glucose uptake (and insulin sensitivity) may be enhanced by leptin in the presence of normal levels of insulin and glucagon. $^{24}$

Contrasting results have also been observed in some studies in which administration of leptin decreased insulin levels. In our study, the administration of leptin caused a reduction in the blood glucose levels which might be attributed to the above mentioned ability of leptin to enhance glucose uptake by the skeletal muscles as well as the insulin sensitising action of leptin.

When the alternation rates (Table 3) were compared between the 3 groups it was found that the alternation rate of the control group (group 1) was the highest at $84 \%$. The alternation rate of the group 3 (diabetic rats that had received leptin) was $65 \%$, which was more than that of the group 2 rats, i.e. $57 \%$ but less than the control rats. This can be explained by the findings of studies done previously that showed that moderate cognition impairment of learning and memory, particularly impairment of spatial learning and memory occurs in diabetic rats. ${ }^{25}$

Alternation reflects the rats' motivation to explore new environments and look out for food shelter etc. This alternation is based on their working memory which in turn is comprised of their short term learning and information gathering tendencies and ability to remember events.

Diabetes causes a dysglycemic picture in which episodes of hyperglycemia alter with episodes of hypoglycaemia. This causes damage to the brain seen as changes in the white matter in various studies. This damage is evident as deficit in the working memory of the rats and can be seen as a reduction in the exploratory tendencies as evident in the $\mathrm{T}$ maze alternation test.

The other parameters that were tested were the time taken to reach the target arms, the time spent immobile by the rodents and the speed of the animal while performing the task. Time taken to complete the task indicated the ability of the animal to take a decision to choose one particular side, its exploratory tendency and motivation to complete the task. Time spent immobile was an indicator of sluggish nature of reflexes and the inability to carry forward with the task of moving around in the maze. Speed is an important parameter to check for the development of cognitive impairment in type 1 diabetes. Cognitive impairment commonly manifests as a reduction in speed of the animal performing the task.

In our study, group 2 rats, i.e. the rats that had received normal saline after becoming diabetic, showed a marked reduction in their performance when compared to the control rats measured by an increase in the time taken to reach the target arms in both the phases of the experiment (Figure 1). This showed a decrease in both the learning ability (as seen in the phase 1) and the memory (as seen in phase 2). The time spent immobile in both the phase 1 and phase 2 increased when compared to the control rats and the speed with which the rats performed the task decreased. All of these findings indicated that cognitive impairment had developed in the diabetic rats.

This impairment can be attributed to the occurrence of hyperglycaemic episodes that might have alternated with hypoglycaemic episodes as well as the direct toxic effects of streptozotocin. Hyperglycemia is not known to cause such dramatic changes in the cognition in such a short duration of 1 month. It might be a contributing reason but not the major cause of the deficits seen in cognition.

On the other hand, the episodic occurrence of hypoglycaemia might be a major contributor to the damage caused to the central nervous system (CNS) in diabetes. Hypoglycaemia causes localised hyperaemia and neurovascular changes which are dependent on neuronal oxygen consumption. ${ }^{26}$ Hypoglycaemia causes glutamate release and activation of neuronal glutamate receptors, production of reactive oxygen species, neuronal zinc release, activation of poly (ADP-ribose) polymerase, and mitochondrial permeability transition all of which cause damage to the CNS.

In a study done by Ghasemi et al results were obtained on similar lines and streptozotocin administration caused an increase in the time taken to find the platform and increase in the escape latency indicating cognitive impairment. $^{12}$

Leptin administration to the group 3 rats in our study brought about a decrease in the time taken to reach the target arm in both the phases (1 and 2), decreased the time spent immobile (Figure 2) and increased the speed of the rodent when compared to the group 2 rats, however the changes were not statistically significant (Figure 3 ). When compared to the control rats (group 1), the time taken to reach the target and the time spent immobile were more. The speed with which the animals performed the task was also less when compared to the group 1 control rats.

The inference that can be drawn from these results is that while leptin administration produced an improvement in the cognitive assessment parameters in the diabetic rats when compared to the diabetic controls, the improvement was not so pronounced as to be comparable with the group 1 non diabetic rats indicating a modest improvement in cognition.

In the experiment performed by Ghasemi et al, administration of leptin produced improvement in learning and memory functions. ${ }^{12}$ Leptin in their study also produced a reduction in the glucose levels which was parallel to the cognitive improvement seen in the diabetic rats. Our study findings seemed to be consistent with this study. 
Leptin is known to increase the sensitivity to insulin however its effects on increasing or decreasing insulin levels have not been clearly known with some studies revealing an increase in insulin production post leptin administration as some demonstrating a decrease in insulin levels. Leptin by its effect on glucose homeostasis wherein it increases the glucose uptake by the peripheral tissues, reduces glucose production by the liver, reduces food intake by acting on the satiety centre, and normalises glucagon levels. ${ }^{27}$ The mechanism by which leptin does this is complex and is multifactorial.

The action of leptin on JAK-STAT pathway, on the Phosphatidylinositol-4,5-bisphosphate 3-kinasepathway in the hypothalamus, on the arcuate nucleus and the proopiomelanocortin cells which bring about the actions of leptin are all responsible for the glucose homeostasis. Leptin acts on the NMDA receptors, the ATP-sensitive $\mathrm{K}^{+}$channels in the hypothalamus and $\mathrm{Ca}^{2+}$-activated $\mathrm{K}^{+}$ channels in the hippocampus bringing about the regulation of neuronal excitability. ${ }^{28}$

Leptin is known to have neurogenetic potential by causing synaptic modulation and dendritic growth and facilitates the conversion of short term potentiation into long term potentiation. $^{29}$ Leptin also puts a brake on AMPK overactivity which is essential in its cognition improving effect. $^{30}$

Leptin administration in congenital leptin deficiency has proven to be beneficial in clinical studies. It has decreased insulin levels, increased insulin sensitivity, lowered levels of triglycerides while improving HDL levels. Effects on thyroid hormone levels, immune function have also been reported. ${ }^{31}$ Its use as a magic bullet for weight loss has been sparked after multiple clinical trials showing benefit in obesity. However this weight loss isn't sustained for a long period of time and hence brings about the idea that the weight loss brought about by leptin can have multiple factors playing role in synergism. All these roles of leptin can be considered to be contributory to its memory improving action. Leptin in human studies has also shown benefits in cases of mild cognitive impairment wherein it was associated with other metabolic alterations and its circulating serum levels were low.

There is a bridge between the clinical and the experimental studies done to assess the role of leptin in cognition with both the study types pointing out towards the fact that leptin's effect on cognition is an indispensible and yet a less explored arena.

\section{CONCLUSION}

It is concluded that the glucose lowering effect of leptin along with all the above mentioned actions contributed to the benefit observed in the rats. Our study thus showed that leptin administration caused a reduction in the blood glucose levels in the rodents and proved to be beneficial in improving the learning and memory of the rats. The mechanism of action by which leptin brought these improvements is multifactorial and needs to be elucidated in further studies.

\section{ACKNOWLEDGEMENTS}

We are thankful to Dean Dr. Jayesh M. Brahmbhatt and Institutional Animal Ethics Committee (IAEC), Government Medical College, Surat, Gujarat, India for giving permission to carry out this work.

Funding: No funding sources

Conflict of interest: None declared

Ethical approval: The study was approved by the Institutional Animal Ethics Committee (IAEC), Government Medical College, Surat, Gujarat, India

\section{REFERENCES}

1. Taranum A, Shakeel M. A Study of Frequency and Factors Associated with Depression among Adult Diabetics in Urban Areas of Davangere, Karnataka. National J Community Med. 2016;7(2):111-5.

2. WHO, Definition and diagnosis of diabetes mellitus and intermediate hyperglycaemia. Available at: http://www.who.int/diabetes/publications/diagnosis_ diabetes2006/en/. Accessed on 3 October 2019.

3. http://www.who.int/features/factfiles/diabetes/en/

4. Gispen W, Biessels G. Cognition and synaptic plasticity in diabetes mellitus. Trends Neurosci. 2000;23(11):542-9.

5. Kalra S. Cognitive impairment and diabetes. Recent Pat Endocr Metab Immune Drug Discov. 2013;7(2):155-65.

6. Cheng C, Tsai Y, Tsai C, Chou P, Lan T. Type 2 diabetes and anti diabetic medications in relation to dementia diagnosis. J Gerontol A Biol Sci Med Sci. 2014;69:1299-305.

7. Brands A, de Haan E, Kappelle L, Kessels R: The Effects of Type 1 Diabetes on Cognitive Performance: a meta-analysis diabetes. Care. 2005;28:726-35.

8. McCrimmon R, Frier B. Diabetes and cognitive dysfunction. Lancet. 2012;0140-6736(1012):60360-2.

9. Van Duinkerken E. Cognitive function in diabetes. Diapedia. 2014;6104716156:4.

10. Dayne B, Harvey J. Leptin: A Novel Therapeutic Target in Alzheimer's Disease? Int J Alzheimer's Dis. 2012;594137:7.

11. Keiichi H, Becjer D, Silva A. Leptin Before and After Insulin Therapy in Children with New-Onset Type 1 Diabetes. J Clin Endocrinol Metab. 1999;84(5):1524-6.

12. Ghasemi M, Zendehbad B, Zabihi H, Hosseini M, Hadjzadeh MAR, Hayatdavoudi P, et al. Beneficial Effect of Leptin on Spatial Learning and Memory in Streptozotocin-Induced Diabetic Rats. Balkan Med J. 2016;33:102-7.

13. Kravitz E, Schmeidler J, Schnaider Beeri M. Type 2 diabetes and cognitive compromise: potential roles of 
diabetes-related therapies. Endocrinol Metab Clin North Am. 2013;42(3):489-501.

14. Janson J, Laedtke T, Parisi JE, O'Brien P, Petersen $\mathrm{RC}$, Butler PC. Increased risk of type 2 diabetes in Alzheimer disease. Diabetes. 2004;53(2):474-81.

15. Ravona-Springer R, Luo X, Schmeidler J, Wysocki M, Lesser G, Rapp M, et al. Diabetes is associated with increased rate of cognitive decline in questionably demented elderly. Dement Geriatr Cogn Disord. 2010;29(1):68-74.

16. Brands AMA, Biessels GJ, de Haan EHF, Kappelle LJ, Kessels RPC. The Effects of Type 1 Diabetes on Cognitive Performance. Diabetes Care. 2005;28(3):726-35.

17. Sachon C, Grimaldi A, Digy JP, Pillon B, Dubois B, Thervet F: Cognitive function, insulin-dependent diabetes and hypoglycaemia. $\mathbf{J}$ Intern Med. 1992;231:471-5.

18. Denroche HC, Huynh FK, Kieffer TJ. The Role of Leptin in Glucose Homeostasis. J Diabetes Investigation. 2012;3(2):115-29.

19. Heymsfield SB, Greenberg AS, Fujioka K. Recombinant leptin for weight loss in obese and lean adults: a randomized, controlled, dose-escalation trial. JAMA. 1999;282:1568-75.

20. Soliman AT, Omar M, Assem HM. Type -1 diabetes mellitus: relationship to body mass index, insulin dose, and glycemic control Serum leptin concentrations in children with type. Metabolism. 2002;51:292-6.

21. Berti L, Kellerer M, Capp E, Haring HU. Leptin stimulates glucose transport and glycogen synthesis in $\mathrm{C} 2 \mathrm{C} 12$ myotubes: evidence for a PI3-kinase mediated effect. Diabetologia. 1997;40:606-9.

22. Okada T, Kawano Y, Sakakibara T, Hazeki O, Ui M. Essential role phosphatidylinositol 3-kinase in insulin-induced glucose transport and anti lipolysis in rat adipocytes. Studies with a selective inhibitor wortmannin. J Biol Chem. 1994;269:3568-73.

23. Welsh GI, Foulstone EJ, Young SW, Tavare JM, Proud CG. Wortmannin inhibits the effects of insulin and serum on the activities of glycogen synthase kinase-3 and mitogen-activated protein kinase. Biochem J. 1994;303:15-20.

24. Sarmiento U, Benson B, Kaufman S, Ross L, Qi M, Scully S, DiPalma C. Morphologic and molecular changes induced by recombinant human leptin in the white and brown adipose tissues of C57BL/6 mice. Lab Invest. 1997;77:243-56.

25. Biessels G, Kamal A, Ramakers GM, Urban IJ, Spruijt BM, Erkelens DW, et al. Place Learning and Hippocampal Synaptic Plasticity in StreptozotocinInduced Diabetic Rats. Diabetes. 1996;45:1259-66.

26. Rosenthal J. The Effect of Acute Hypoglycemia on Brain Function and Activation. Diabetes. 2001;50(7):1618-26.

27. Wang MY. Leptin therapy in insulin-deficient type I diabetes. PNAS USA. 2010;107:4813-9.

28. Harvey J. Leptin regulation of neuronal excitability and cognitive function. Curr Opin Pharmacol. 2007;7(6):643-7.

29. Garza JC, Guo M, Zhang W, Lu XY. Leptin increases adult hippocampal neurogenesis in vivo and in vitro. J Biol Chem. 2008;283(26):18238-47.

30. Dagon Y, Avraham Y, Magen I, Gertler A, Ben-Hur T, Berry EM. Nutritional status, cognition, and survival: a new role for leptin and AMP kinase. J Biol Chem. 2005;280(51):42142-8.

31. Farooqi IS, Matarese G, Lord GM. Beneficial effects of leptin on obesity, $\mathrm{T}$ cell hyporesponsiveness, and neuroendocrine/metabolic dysfunction of human congenital leptin deficiency. J Clin Invest. 2002;110(8):1093-103.

Cite this article as: Joshi AC, Patel CR, Kantharia ND. Effect of leptin on spatial learning, memory and blood glucose level in streptozotocin induced diabetes mellitus in male wistar albino rats. Int $\mathbf{J}$ Basic Clin Pharmacol 2020;9:24-31. 